\title{
Choice Behavior of the Elderly Regarding Street-Crossing Facility
}

\author{
Chong Wei (iD), Xuedong Yan (iD, Chunfu Shao, Jiang Sun, and Lu Ma (iD) \\ MOE Key Laboratory for Urban Transportation Complex Systems Theory and Technology, School of Traffic and Transportation, \\ Beijing Jiaotong University, Beijing 100044, China
}

Correspondence should be addressed to Chong Wei; chwei@bjtu.edu.cn

Received 15 August 2017; Revised 23 November 2017; Accepted 14 December 2017; Published 4 January 2018

Academic Editor: Ángel Ibeas

Copyright (C) 2018 Chong Wei et al. This is an open access article distributed under the Creative Commons Attribution License, which permits unrestricted use, distribution, and reproduction in any medium, provided the original work is properly cited.

This study aims to understand the street-crossing-facility choice behavior of the elderly. The footbridges were classified into three types with different levels of convenience. On the other hand, the safety of the crosswalk was assumed to be highly related to the remaining time of pedestrian green light. An adaptive SP survey was designed to collect choice data from individuals aged 60 years or above, and a multilevel logistic model was developed to analyze the behavior data. The results indicated that convenience and safety exert significant influence on the facility choice behavior. The choice behavior of the elderly toward making trade-off decision between convenience and safety was further discussed through the multilevel logistic regression analyses.

\section{Introduction}

As individuals age, fewer journeys are undertaken as car drivers and a higher number of journeys are undertaken as pedestrians (Dunbar et al. 2004). Owing to age-related decline, elderly pedestrians could encounter higher safety challenges while crossing streets. For example, Asher et al. [1] surveyed a random sample of older people in the general population in England to obtain elderly pedestrians' normal walking speed. It was determined that, among individuals aged over 65 years in England, 84\% of male pedestrians and $94 \%$ of female pedestrians were incapable of crossing roads in time. Kong et al. [2] conducted a computer simulation to investigate whether the duration of pedestrian green signal could ensure elderly pedestrians to complete street-crossing. The result indicated that above $50 \%$ of the subjects older than 70 years could not cross streets in time. Dommes et al. [3] concluded that slow decision-making, decreased walking speed, and the challenges in selecting safe gaps and adopting sufficient safety margins cause the elderly to encounter higher hazards when they cross streets. These studies indicate that the risk involved in street-crossings by the elderly is considerable. Therefore, it is worthwhile to further investigate the underlying street-crossing behavior of the elderly in order to reduce the risk associated with such street-crossings.
This study attempted to analyze the street-crossingfacility choice problem encountered by the elderly in terms of behavior analyses. Certain street-crossing facilities, such as footbridge, can provide a high safety level for elderly pedestrians by physically separating the pedestrian flow from the vehicular traffic. However, the inconveniences of longer travel distance contributed by up-down movements and of higher body moments requirement may prevent elderly pedestrians from using them. Elder pedestrians are required to make a trade-off choice between safety and convenience. The street-crossing facilities considered in this study were footbridges and signalized crosswalks with countdown timer (unsignalized crosswalks and pedestrian underpasses are unusual in Beijing). Equipping footbridges with escalators can reduce their inconvenience [4]. The footbridges were further classified into three types in this study: (I) without escalators, (II) with escalators in one direction, and (III) with escalators in both up and down directions. For the signalized pedestrian crosswalks, the remaining time of pedestrian green light is related to the safety of street-crossing [1], where a longer remaining time can result in a higher safety level of pedestrians.

A number of studies have indicated that the perceived safety and convenience can significantly impact streetcrossing behavior. For example, Havard and Willis [5] demonstrated that installing a marked crosswalk can enhance 
the street-crossing experience of pedestrians because individuals felt safer when crossing the road after the zebra-crossing had been installed. Kadali and Vedagiri (2015) and Bian et al. (2014) confirmed that pedestrians' level of service (LOS) at crosswalks was strongly dependent on perceived safety. Keegana and O'Mahony (2003) investigated the reasons for pedestrians waiting at pedestrian lights and determined "danger/fear/safety" to be the primary reason. On-street pedestrian surveys by Fitzpatrick et al. (2004) indicated that selection of pedestrian-crossing location was highly dependent on pedestrians' perception of safety. Compared to crosswalks, a footbridge allows individuals to feel safer. However, previous studies observed that footbridges failed to attract pedestrians owing to the inconvenience of utilization. Jensen (1998) [6] and Zegeer (2002) indicated that long crossing distances and steep slopes can discourage pedestrians from using footbridges. Räsänen et al. [4] studied the pedestrians' preferences for footbridges through self-report analyses and identified the effects of the footbridges' physical features such as the width of bridge and the number of steps to be walked in the footbridges. The study also reported that escalators exhibit the potential to attract pedestrians to use footbridges. Anciaes and Jones [7] report the results of a study to understand the preferences of pedestrians toward different types of crossing facilities, but their study ignores the effect of the remaining time of green light.

It is worthwhile to note that previous studies generally considered the utilization of crossing facilities (e.g., crosswalks and footbridges) separately through field survey data; therefore, there is still a requirement for investigating elderly pedestrians' behavior toward the decision between footbridges and crosswalks. To further explore the elderly pedestrian's underlying crossing-facility selection behavior, we conducted an SP survey for collecting corresponding behavior data. We recognized that the attributes of streetcrossing facilities can be rather involved for the elderly to understand when described by textual representation. Therefore, the traditional paper-based SP survey may not effectively collect street-crossing-facility selection behavior data, particularly if the responders of the survey are elderly. On the other hand, a computer-based SP survey could display attributes of alternatives through multimedia such as videos and $3 \mathrm{D}$ images. Multimedia provides a straightforward method to allow responders to accurately understand the features of alternative facilities and thus reduce perception bias. A few researchers have already adopted computer-based SP surveys to collect travel behavior data. For example, Tilahun et al. [8] used a group of video clips to display the cycle lane environment to SP survey responders; Iryo et al. [9] used a virtual-reality (VR) environment to present the scenarios of an SP survey to collect pedestrian's route selection behavior data. Kang et al. (2013) adopted video clips to present Chinese urban sidewalks to responders in an SP survey for collecting behavior data about pedestrian perceptions of level of service. Domes et al. (2014) conducted a simulated street-crossing experiment to investigate the street-crossing decision and behavior of older and younger pedestrians. These studies provided effective case studies on the role of emerging technologies in enhancing the
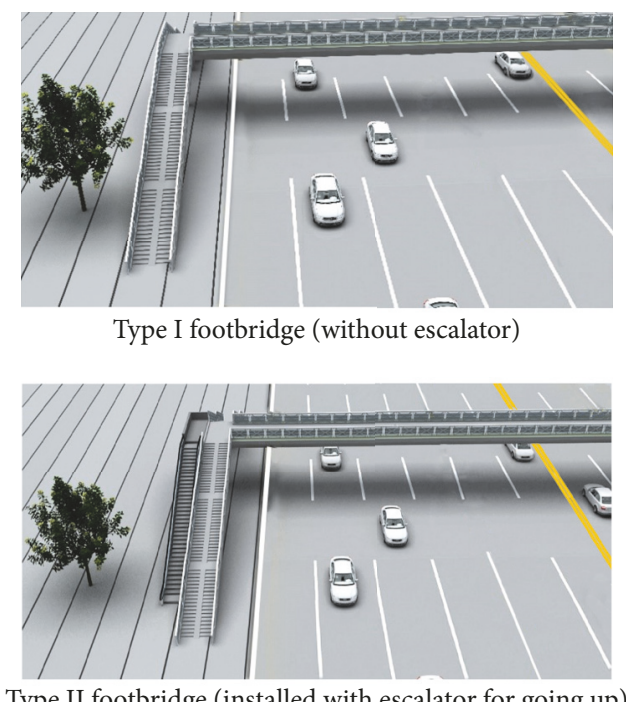

Type II footbridge (installed with escalator for going up)

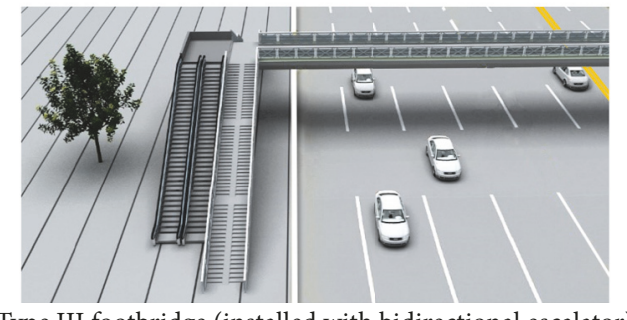

Type III footbridge (installed with bidirectional escalator)

FIgURE 1: Type I, type II, and type III footbridges.

performance of SP survey. However, few studies discuss the approach to appropriately represent lengths of time such as remaining time of pedestrian green light. To collect elder pedestrian's street-crossing behavior data with high quality, it is necessary to resolve this problem effectively.

This study focused on elderly pedestrians' street-crossing behavior and investigated a key problem that has not been discussed in the previous studies. We attempted to understand the behavior of elderly pedestrians toward trade-off decisions between safety and convenience when they choose various types of street-crossing facilities. The remaining paper is organized as follows: Section 2 describes the background of the study. Section 3 describes our design of an adaptive SP survey for collecting data on selection of streetcrossing facility; in order to implement the survey effectively, we developed a novel method to represent the remaining time of pedestrian green light through video editing technology. Section 4 describes our employment of a multilevel logistic regression model to analyze the collected data in order to measure the effects of safety and convenience factors on the utilization of street-crossing facilities. Section 5 offers a discussion and concludes the study.

\section{Background}

We examined three types of footbridges (types I, II, and III), as shown in Figure 1. Type I footbridge incorporates stairs and not an escalator; type II footbridge incorporates stairs and an 
TABLE 1: An example of survey questions.

\begin{tabular}{lcccc}
\hline Responder ID & Question number & Footbridge & Remaining time (s) & Choice result \\
\hline 1 & 1 & Type I & 32 & Crosswalk \\
1 & 2 & Type I & 39 & Footbridge \\
1 & 3 & Type II & 37 & Crosswalk \\
1 & 4 & Type II & 37 & Footbridge \\
1 & 5 & Type III & 40 & Footbridge \\
1 & 6 & Type III & 31 & Crosswalk \\
2 & 1 & Type I & 28 & Footbridge \\
2 & 2 & Type I & 33 & Footbridge \\
2 & 3 & Type II & 36 & Crosswalk \\
2 & 4 & Type II & 36 & Footbridge \\
2 & 5 & Type III & 39 & Crosswalk \\
2 & 6 & Type III & & \\
\hline
\end{tabular}

escalator for going up; type III footbridge incorporates stairs and escalators for going up and down. The inconvenience of a footbridge can be reduced by installing escalators. On the other hand, the safety risk in the crosswalks is dependent on the remaining time of pedestrian green light. The shorter the remaining time of the pedestrian green light is, the higher the risk involving elderly pedestrians is.

We were mainly interested in understanding the effects of installation of escalator (corresponding to convenience) and of the length of remaining time of pedestrian green light (corresponding to safety) on street-crossing-facility choice behavior. Thus, the experiment scenario for street-crossingfacility choice was designed as follows: the locations of footbridges are as close to crosswalks as feasible in order that the distance between a footbridge and a crosswalk can be omitted in the behavior analysis. To prevent unnecessary complexity in the pedestrian light setting scenario, this study did not consider the waiting time owing to pedestrian red light and illegal street-crossing behavior is also beyond the scope of this research.

\section{Adaptive SP Survey Design and Implementation}

3.1. Survey Design. An adaptive SP survey was conducted to collect the data related to street-crossing behavior. In the survey, it was assumed that the width of the street that pedestrians were required to cross was $32 \mathrm{~m}$. Accordingly, the remaining time of pedestrian green light was set within the range from $26 \mathrm{~s}$ to $52 \mathrm{~s}$ (Knoblauch et al. [10] reported that average speeds for the elderly have been estimated around $1.25 \mathrm{~m} / \mathrm{s}$; however, pedestrians have to pay attention to turnleft/right cars when they cross a street, so the average speed for street-crossing is smaller than the normal walking speed). We considered that the escalator's speed and the length of the foot bridge are constant in the survey and the values about the speed and the length are not involved in the regression model. Throughout the survey procedure, each responder was asked six questions. In each question, a type of bridge and a scenario of the remaining time of pedestrian green light were displayed. The first and second questions involved the type I bridge, the third and fourth questions involved the type II bridge, and the last two questions involved the type III bridge. Table 1 provides an example.

The remaining time of pedestrian green light in each question was adjusted according to the response to the previous question. For the $i$ th question, we undertook adjustments according to the following rules:

(1) In the cases where $i=1$, we randomly selected a length of the remaining time of pedestrian green light from the range from $26 \mathrm{~s}$ to $52 \mathrm{~s}$.

(2) In the cases where $i=2,4,6$, we deducted $3 \mathrm{~s}$ from the remaining time in the $i$ th question if the responder selected the crosswalk in the $(i-1)$ th question; otherwise, we added $3 \mathrm{~s}$ to the remaining time of pedestrian green light for the $i$ th question.

(3) In the cases where $i=3,5$, we did not adjust the remaining time for the question if the choice result in the $(i-1)$ th question was crosswalk; otherwise, we added $3 \mathrm{~s}$ to the remaining time of pedestrian green light.

(4) We limited the remaining time of pedestrian green light to the range from $26 \mathrm{~s}$ to $52 \mathrm{~s}$. If the rule required the remaining time in the $i$ th question to be adjusted to more than $52 \mathrm{~s}$, then we set the remaining time to $52 \mathrm{~s}$ for the $i$ th question; on the other hand, if the rule required the remaining time to be adjusted to less than $26 \mathrm{~s}$, we set the remaining time to $26 \mathrm{~s}$ for the $i$ th question.

For each question, we used a sequence of $3 \mathrm{D}$ computer images to display the attributes of the footbridge; we selected eight images for eight points of view and displayed these images to a responder one by one. We did not use actual photographs to display footbridges because the background and the shooting angle of the photographs can impact the behavior survey and raise bias. On the other hand, 3D computer images permit us to maintain a consistent background in all the images and provide a straightforward method to present a footbridge from various points of view. 


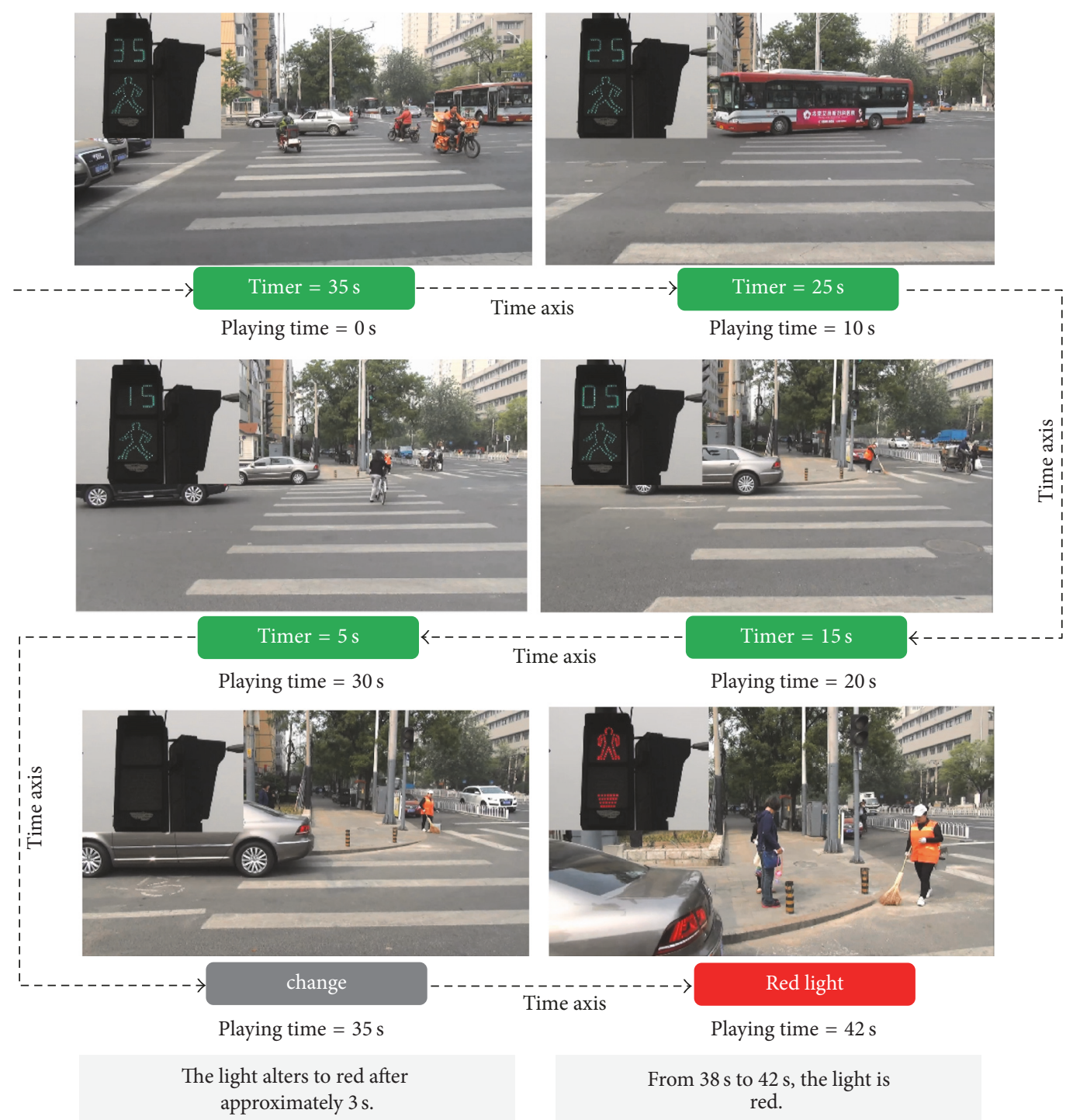

FIGURE 2: Synthetic video: the time remaining for the pedestrian green light is $35 \mathrm{~s}$.

We used a synthetic video to enable responders to experience the remaining time of pedestrian green light (Figures 2 and 3) setting in the question. The synthetic video displayed a crosswalk with a countdown timer. At the beginning of the video, the remaining time of the pedestrian green light displayed by the countdown timer was equal to the remaining time of pedestrian green light set in the question. The remaining time displayed by the countdown timer decreased as the video was played. The video used a long, lingering shot to present the first-person view of an individual who walked from the origin to the destination of the crosswalk.

Figure 2 displays a series of frames of synthetic video, where the corresponding remaining time of pedestrian green light was $35 \mathrm{~s}$. At the $35 \mathrm{~s}$ point in the video, the traffic light did not display anything and altered to red after approximately 3 s. From 38 s to $42 \mathrm{~s}$, the traffic light was red.

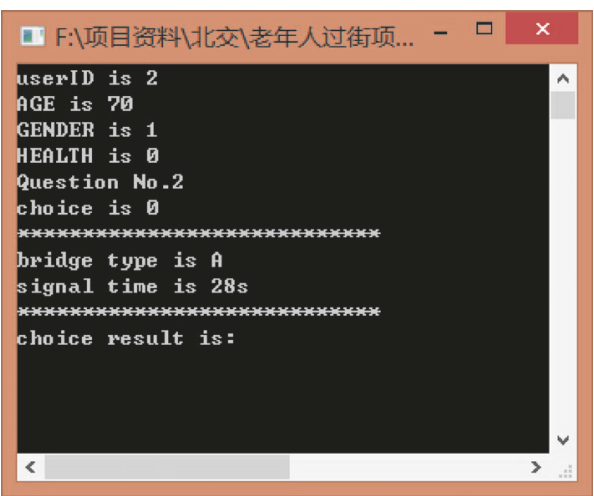

FIGURE 3: The command line tool that is developed for managing the survey and generating questions (in the screen shot " $\mathrm{A}$ " indicates type I footbridge). 
TABLE 2: Responders from four communities.

\begin{tabular}{|c|c|c|c|c|}
\hline \multirow{2}{*}{ Community } & \multirow{2}{*}{ Number of participants } & \multicolumn{2}{|c|}{ Gender distribution } & \multirow{2}{*}{ Average age (year) } \\
\hline & & Male & Female & \\
\hline Beijiaoda & 40 & $27 \%$ & $73 \%$ & 70.1 \\
\hline Zaojundongli & 50 & $38 \%$ & $62 \%$ & 65.4 \\
\hline Zaojunxili & 44 & $34 \%$ & $66 \%$ & 67.0 \\
\hline Tiekeyuan & 35 & $25 \%$ & $75 \%$ & 74.5 \\
\hline
\end{tabular}

We developed a command line tool to manage the SP survey (see Figure 3). It is impractical to let the elderly operate a computer to answer questions. Throughout the survey procedure, we told the responders that the trip in the survey is a regular trip. Our staffs helped responders to input the choice result of a question into the command line tool so that it can provide the bridge type and scenario of the remaining time of pedestrian green light for the subsequent question. Then, our staff displayed the corresponding images and video to the responder through a laptop. Before asking questions, we also recorded the demographic information such as age and gender of the responder by the command line tool.

3.2. Behavior Data. We enrolled 169 responders aged 60 years or above from four communities located at Haidian district, Beijing (Table 2). The participants are ordinarily resident in these areas. As mentioned above, we asked each responder six questions; each question had two alternatives (footbridge and crosswalk). The intersection presented in the first-person view video is closed to the communities listed by Table 2 .

The adaptive SP survey system can generate questions that are more targeted than random questions. The survey system automatically adjusts the remaining time of green light according to the answer of the last question. After the survey, we analyzed the attributes of the questions. We grouped the questions by the bridge type and calculated the average remaining time for each group (i.e., for each bridge type). Figure 4 reports the result. We found that the average remaining time for the group of type III footbridge is longer than that of the group of type II footbridge, while type II footbridge also has a longer average remaining time than that of type I footbridge. This result implies that escalators exhibit the potential to attract pedestrians to use footbridges. We provided more detailed analysis by a regression model in Section 4.

\section{Behavior Model}

4.1. Multilevel Logistic Regression Model. In order to consider the heterogeneity among the responders, this study employed a multilevel logistic model to analyze the behavior data collected by the adaptive SP survey. The model can be formulated as follows:

$$
\begin{aligned}
U_{i k}= & V_{i k}+\varepsilon_{i}+\tau_{i k} \\
= & \alpha+\beta_{A} \cdot A_{i}+\beta_{G} \cdot G_{i}+\beta_{O} \cdot O_{i k}+\beta_{D} \cdot D_{i k}+\beta_{T} \\
& \cdot T_{i k}+\varepsilon_{i}+\tau_{i k},
\end{aligned}
$$

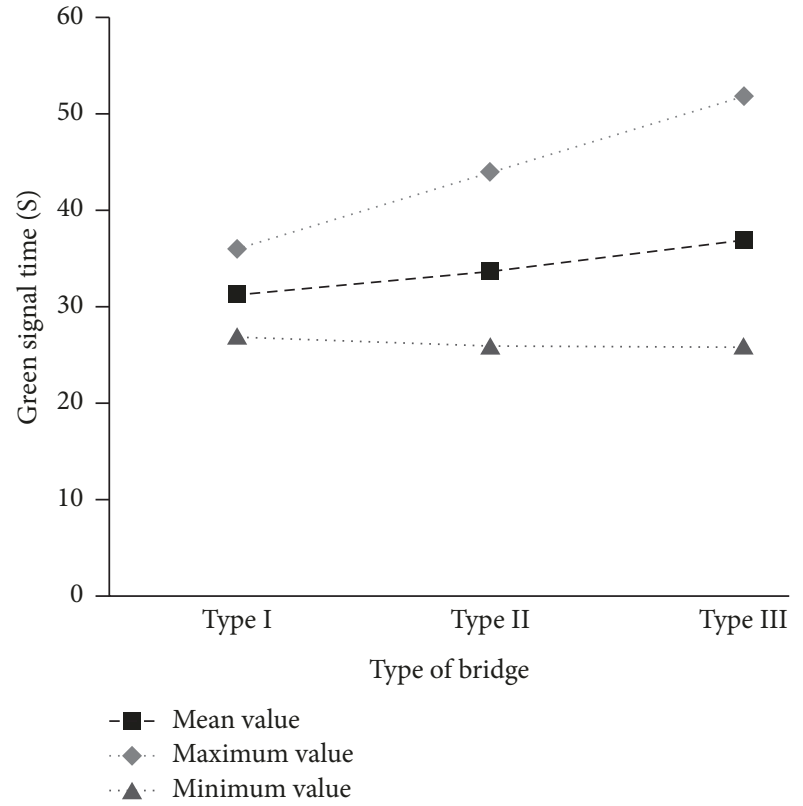

FIGURE 4: Characteristics of the remaining time of pedestrian green light.

where

$$
\begin{aligned}
\tau_{i k} & \sim \operatorname{Logistic}(0,1) \\
\varepsilon_{i} & \sim N\left(0, \sigma^{2}\right), \\
Y_{i k} & = \begin{cases}1 & \text { if } U_{i k}>0 \\
0 & \text { otherwise }\end{cases}
\end{aligned}
$$

where $i$ indicates the $i$ th responder, $k$ indicates the $k$ th question for the responder, and $Y_{i k}$ indicates the selection result (footbridge $=1$; crosswalk $=0$ ). We considered five explanatory variables in this study:

$A_{i}$ : indicating whether the age of responder is higher than or equal to 80 (yes $=1$, no $=0$ )

$G_{i}$ : gender $($ male $=1$, female $=0)$

$O_{i k}$ : indicating whether the footbridge is equipped with an up-going escalator (yes $=1$, no $=0$ )

$D_{i k}$ : indicating whether the footbridge is equipped with a down-going escalator (yes $=1$, no $=0$ )

$T_{i k}$ : the remaining time of the pedestrian green light. 
TABLE 3: $O_{i k}$ and $D_{i k}$ associated with type of footbridge.

\begin{tabular}{lcc}
\hline Footbridge & $O_{i k}$ & $D_{i k}$ \\
\hline Type I & 0 & 0 \\
Type II & 1 & 0 \\
Type III & 1 & 1 \\
\hline
\end{tabular}

TABLE 4: Demographic information of responders.

\begin{tabular}{lccc}
\hline & All responders & Nontrader & Trader \\
\hline $\begin{array}{l}\text { Number of } \\
\text { observations }\end{array}$ & 169 & 20 & 149 \\
\hline Sex & & & \\
$\quad$ Male (\%) & $32 \%$ & $40 \%$ & $31 \%$ \\
$\quad$ Female (\%) & $69 \%$ & $60 \%$ & $69 \%$ \\
\hline Age & & & \\
Mean & 69.7 & 74.0 & 69.2 \\
SD & 8.2 & 7.6 & 8.1 \\
Maximum & 89 & 86 & 89 \\
Minimum & 60 & 60 & 60 \\
\hline
\end{tabular}

The combination of the variables $O_{i k}$ and $D_{i k}$ can present the type of footbridge in the model. Table 3 illustrates the values of the variables corresponding to each type of footbridge.

4.2. Preanalysis. In this study, the choice behavior is described by a utility function with a linear structure. The structure assumes that individuals make a trade-off between the attributes when taking a decision. On the other hand, certain behavior traits that are inconsistent with the assumption of the specified model are likely be observed in the SP survey. Hess et al. [11] recommended using a preanalysis procedure to identify these behavior traits and cleaning the SP dataset to reduce the analysis bias. This study considered a behavior trait that generally arises in SP surveys, namely, nontrading. Responders who are nontraders have a definite preference for alternative and would always choose that alternative regardless of the situations of selection sets.

We identified the nontrader by the following procedure: responder $i$ is a nontrader if $Y_{i k}=1$ for $k=1, \ldots, 6$ or $Y_{i k}=0$ for $k=1, \ldots, 6$. As presented in Table 4, we found 20 responders to be nontraders (they all have $Y_{i k}=0$ for $k=1, \ldots, 6)$. The average age of nontrader is 74.1 , and the average age of trading responders is 69.2.

We conducted an ANOVA to analyze the variation between traders and nontraders. The result of the ANOVA indicated that the mean age of responders with nontrading is significantly higher than that of responders with trading (see Table 5).

4.3. Model Estimation. The parameters of the multilevel logistic model were estimated using the behavior data after removing the responses by the nontrading responders. We used MCMCglmm package of $\mathrm{R}$ project to carry out the estimation [12].
Table 6 displays the estimated result for the multilevel logistic model expressed by (1). We observed that the parameter before $G_{i}$ (gender) is not significant (the sign for the parameter indicates that males exhibit a tendency to choose crosswalk to cross streets).

We modified the regression model by removing variable $G_{i}$ from the linear function as illustrated by the following equation:

$$
\begin{aligned}
U_{i k}= & \alpha+\beta_{A} \cdot A_{i}+\beta_{O} \cdot O_{i k}+\beta_{D} \cdot D_{i k}+\beta_{T} \cdot T_{i k}+\varepsilon_{i} \\
& +\tau_{i k} .
\end{aligned}
$$

We repeated the estimation using MCMCglmm. Table 7 presents the estimated result of the modified model. The sign of $\beta_{A}$ is negative, which implies that responders of age above 80 years exhibit a low tendency to choose footbridges. Table 7 also reveals that $\beta_{T}$ is significant $(p$ value $<0.001)$ and is negative. This result indicates that the remaining time of pedestrian green light exerts a negative impact on the selection probability of footbridge. As the remaining time of pedestrian green light is related to the level of safety, this result also implies that individuals exhibit a low tendency to use footbridges if they consider the crosswalks to be safe. Variables $O_{i k}$ and $D_{i k}$ respect escalator equipping for footbridge. The estimated results of $\beta_{O}$ and $\beta_{D}$ reveal that both $O_{i k}$ and $D_{i k}$ are significant $(p$ value $<0.001)$ and positive. This result shows that installing escalator in footbridge can attract a higher number of individuals to use the footbridge. We also observed that the absolute value of $\beta_{O}$ is larger than that of $\beta_{D}$.

The estimation result of the logistic model implies that if we install bidirectional escalators in a footbridge, the crosswalk requires a longer remaining time for pedestrian, that is, an additional $10.23 \mathrm{~s}$, for it to be preferable (note that $\left.\left|\beta_{O} / \beta_{T}\right|+\left|\beta_{D} / \beta_{T}\right|=10.23\right)$.

4.4. Extension. The parameters presented by Table 7 could be used to analyze the street-crossing behavior for a street with a different width. The utility function can be rewritten as follows:

$$
V_{i k}=\alpha+\beta_{A} \cdot A_{i}+\beta_{O} \cdot O_{i k}+\beta_{D} \cdot D_{i k}+\beta_{R} \cdot R_{i k} .
$$

We define $R_{i k}$ as the ratio of the remaining time of the pedestrian green light to the average walking time for older pedestrians to cross the street. Let $v$ denote the average speed of older pedestrians crossing a street. Now, we can obtain the average walking time for older pedestrian to cross the street as $32 / \nu$ (the width of the street in the SP survey is $32 \mathrm{~m}$ ). If we assume $\beta_{R} \cdot R_{i k}$ to be equivalent to $\beta_{T} \cdot T_{i k}$, we can further obtain

$$
\beta_{R}=\frac{32 \beta_{T}}{v}=-\frac{7.14}{v} .
$$

Now, let us consider a street of width of $42 \mathrm{~m}$ as an example. For this case, the contribution of $T_{i k}$ to $V_{i k}$ can be estimated by the following equation:

$$
\beta_{R} \cdot R_{i k}=\left(-\frac{7.14}{v}\right) \cdot\left(\frac{T_{i k} v}{42}\right)=-0.17 T_{i k}
$$


TABLE 5: ANOVA analysis for trader and nontrader.

\begin{tabular}{lccc}
\hline Source of variance & Sum of square & Degree of freedom & Mean square \\
\hline Between & 411.11 & 1 & 411.11 \\
Within & 10774.64 & 167 & 64.52 \\
\hline Total & 11185.75 & 168 & \\
\hline
\end{tabular}

TABLE 6: Estimated result for the multilevel logistic model.

\begin{tabular}{|c|c|c|c|c|}
\hline & Post mean & $I-95 \% \mathrm{CI}$ & $U-95 \mathrm{CI}$ & $p$ value \\
\hline$\beta_{A}$ (age) & -0.75 & -1.70 & 0.14 & 0.09 \\
\hline$\beta_{G}$ (gender) & -0.31 & -0.95 & 0.26 & 0.31 \\
\hline$\beta_{O}$ (going up) & 1.33 & 0.76 & 1.90 & $<0.001$ \\
\hline$\beta_{D}$ (going down) & 1.10 & 0.54 & 1.69 & 0.002 \\
\hline$\beta_{T}$ (time) & -0.25 & -0.40 & -0.11 & $<0.001$ \\
\hline DIC & 1153.22 & & & \\
\hline Number of responders & 149 & & & \\
\hline Number of observations & 894 & & & \\
\hline
\end{tabular}

TABLE 7: Estimated result for the modified model.

\begin{tabular}{|c|c|c|c|c|}
\hline & Post mean & $I-95 \% \mathrm{CI}$ & U-95 CI & $p$ value \\
\hline$\beta_{A}($ age $)$ & -0.73 & -1.71 & 0.08 & 0.084 \\
\hline$\beta_{O}$ (going up) & 1.27 & 0.71 & 1.79 & $<0.001$ \\
\hline$\beta_{D}$ (going down) & 1.01 & 0.35 & 1.56 & $<0.001$ \\
\hline$\underline{\beta_{T}}$ (time) & -0.22 & -0.36 & -0.06 & $<0.001$ \\
\hline DIC & 1161.22 & & & \\
\hline Number of responders & 149 & & & \\
\hline Number of observations & 894 & & & \\
\hline
\end{tabular}

where $T_{i k} \nu / 42$ is the ratio of the remaining time of pedestrian green light to the average walking time for older pedestrians crossing the street of $42 \mathrm{~m}$ width.

\section{Discussion and Conclusions}

In this study, the values of the parameters $\beta_{O}, \beta_{D}$, and $\beta_{T}$ in the regression model permit us to further measure the effect of installing escalator and of the remaining time length of pedestrian green light. We observed that installing escalators to footbridges can significantly enhance the preference of a majority of elderly pedestrians for footbridges. The escalator can weaken pedestrians' motivation for using crosswalks if the remaining length of pedestrian light is not adequate. This fact implies that equipping escalators to footbridges is capable of enhancing the utilization of footbridge as well as enhancing the safety situation for the crosswalk.

In addition to the attributes of street-crossing facilities, the effect of personal attributes on the choice behavior was also investigated. It was determined that age exerted a negative effect on the utilization of footbridge; however, the gender exerted no significant impact on the selection decision. The age factor is also related to whether the responders are willing to make a trade-off decision between safety and convenience. The result revealed that a section of the elderly pedestrians did not make the trade-off choice decision. These responders consistently selected crosswalks; their choice decisions were independent of the attributes of the crossing facilities. Interestingly, these responders' average age is significantly higher than that of the responders who were willing to make the trade-off decisions.

Data collection is a critical issue for choice behavior analysis. This study indicated that the synthetic video can assist researchers in collecting data effectively. The synthetic video provided an effective way to represent temporalspatial attributes of street-crossing facilities. The responders can undergo realistic experience of the remaining time of pedestrian green light by watching the videos.

In future studies, it is recommended to include additional factors into the analysis. We will further consider the waiting time for traffic signal and the distance between the footbridge and the desired crossing location (see Zegeer, 2002) in future studies. Pedestrian behavior is also subject to weather conditions and traffic volume (e.g., Jensen, 1998; Houten et al., 1999); therefore, the corresponding attributes should be incorporated in the SP survey. Further behavior data on young and middle-aged individuals are to be collected 
in order to understand the heterogeneous behavior among various age groups.

\section{Conflicts of Interest}

The authors declare that they have no conflicts of interest.

\section{Acknowledgments}

This work is supported by the National Natural Science Foundation of China (no. 51408035 and no. 71621001) and Beijing Social Science Foundation (no. 16GLB013).

\section{References}

[1] L. Asher, M. Aresu, E. Falaschetti, and J. Mindell, "Most older pedestrians are unable to cross the road in time: a crosssectional study," Age and Ageing, vol. 41, no. 5, pp. 690-694, 2012.

[2] P. W. Kong, Y. K. Chua, C. Y. Ooi, and Y. J. Sim, "Challenges of adequate road crossing time for older persons: A pilot study in Singapore," Journal of the American Geriatrics Society, vol. 61, no. 7, pp. 1226-1227, 2013.

[3] A. Dommes, V. Cavallo, and J. Oxley, "Functional declines as predictors of risky street-crossing decisions in older pedestrians," Accident Analysis \& Prevention, vol. 59, pp. 135-143, 2013.

[4] M. Räsänen, T. Lajunen, F. Alticafarbay, and C. Aydin, "Pedestrian self-reports of factors influencing the use of pedestrian bridges," Accident Analysis \& Prevention, vol. 39, no. 5, pp. 969973, 2007.

[5] C. Havard and A. Willis, "Effects of installing a marked crosswalk on road crossing behaviour and perceptions of the environment," Transportation Research Part F: Traffic Psychology and Behaviour, vol. 15, no. 3, pp. 249-260, 2012.

[6] R. C. Munoz-Raskin and A. Sarasti, "Footbridges or "foolbridges"? analysis of efficacy of three pedestrian bridges as a tool for road safety in Panama city," in Proceedings of the Transportation Research Board 87th Annual Meeting Compendium of Papers DVD, 2008.

[7] R. Anciaes P and P. Jones, "Estimating preferences for different types of pedestrian crossing facilities," Transportation Research F: Psychology and Behavior, 2017.

[8] N. Y. Tilahun, D. M. Levinson, and K. J. Krizek, "Trails, lanes, or traffic: valuing bicycle facilities with an adaptive stated preference survey," Transportation Research Part A: Policy and Practice, vol. 41, no. 4, pp. 287-301, 2007.

[9] T. Iryo, M. Asano, S. Odani, and S. Izumi, "Examining factors of walking disutility for microscopic pedestrian model - a virtual reality approach," Procedia - Social and Behavioral Sciences, vol. 80, pp. 940-959, 2013.

[10] R. L. Knoblauch, M. T. Pietrucha, and M. Nitzburg, "Field studies of pedestrian walking speed and start-up time," Transportation Research Record, no. 1538, pp. 27-38, 1996.

[11] S. Hess, J. M. Rose, and J. Polak, "Non-trading, lexicographic and inconsistent behaviour in stated choice data," Transportation Research Part D: Transport and Environment, vol. 15, no. 7, pp. 405-417, 2010.

[12] MCMCglmm package. https://cran.r-project.org/web/packages/ MCMCglmm/. 


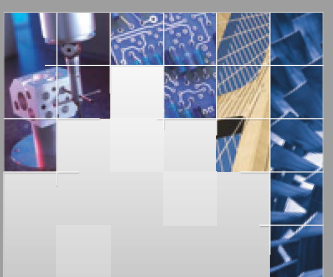

\section{Enfincering}
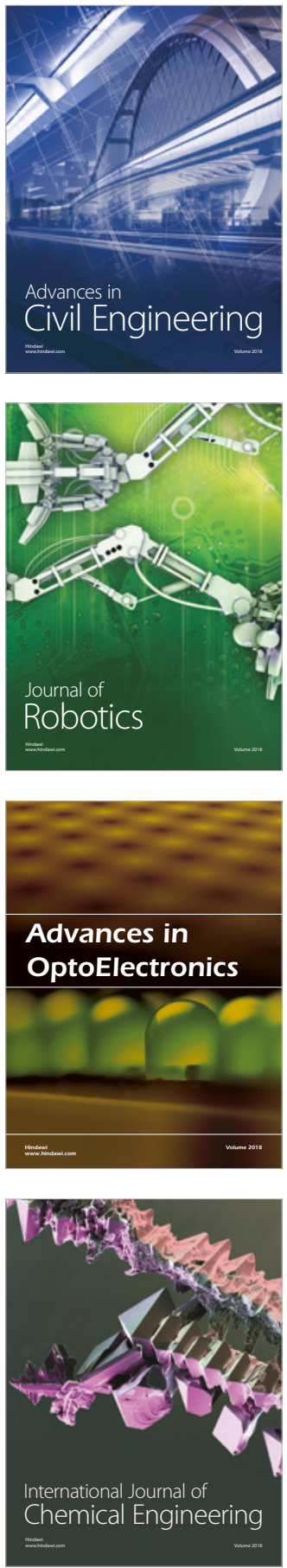

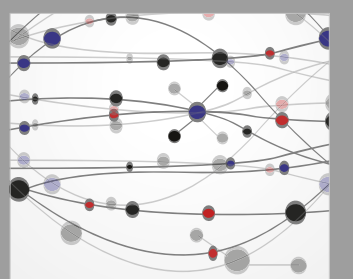

\section{Rotating \\ Machinery}

The Scientific World Journal

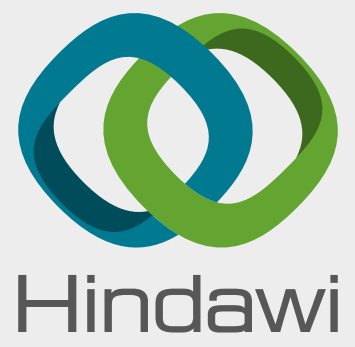

Submit your manuscripts at

www.hindawi.com
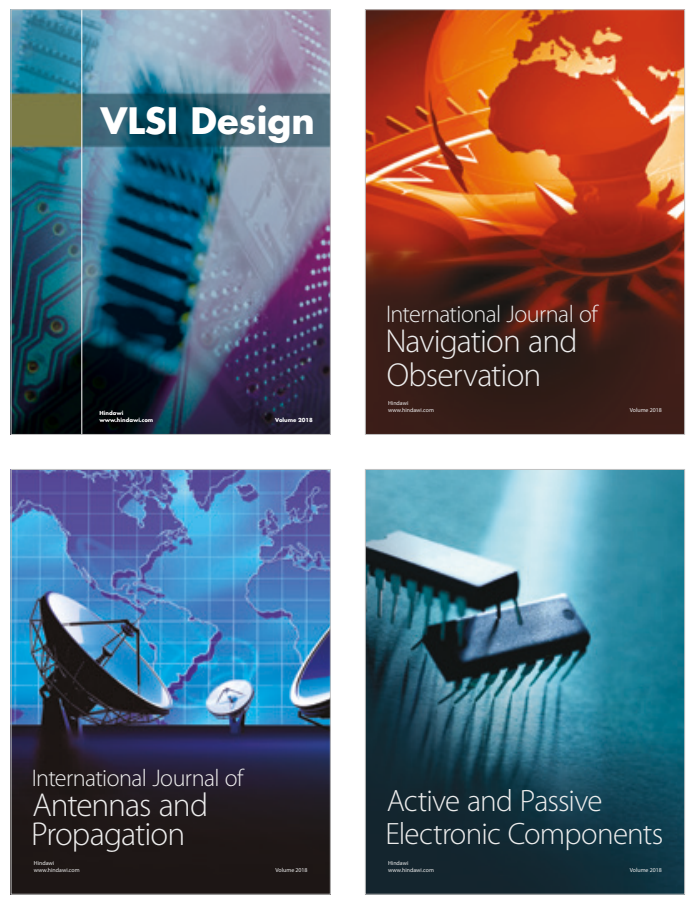
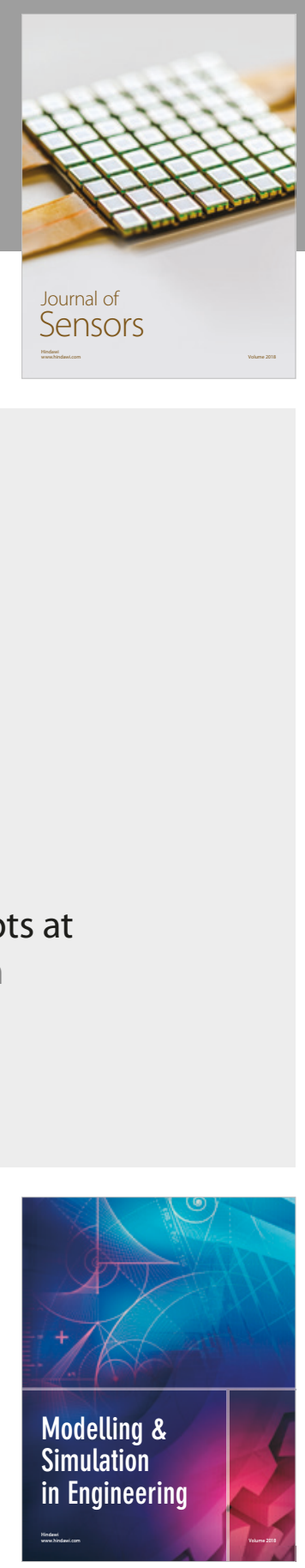

\section{Advances \\ Multimedia}
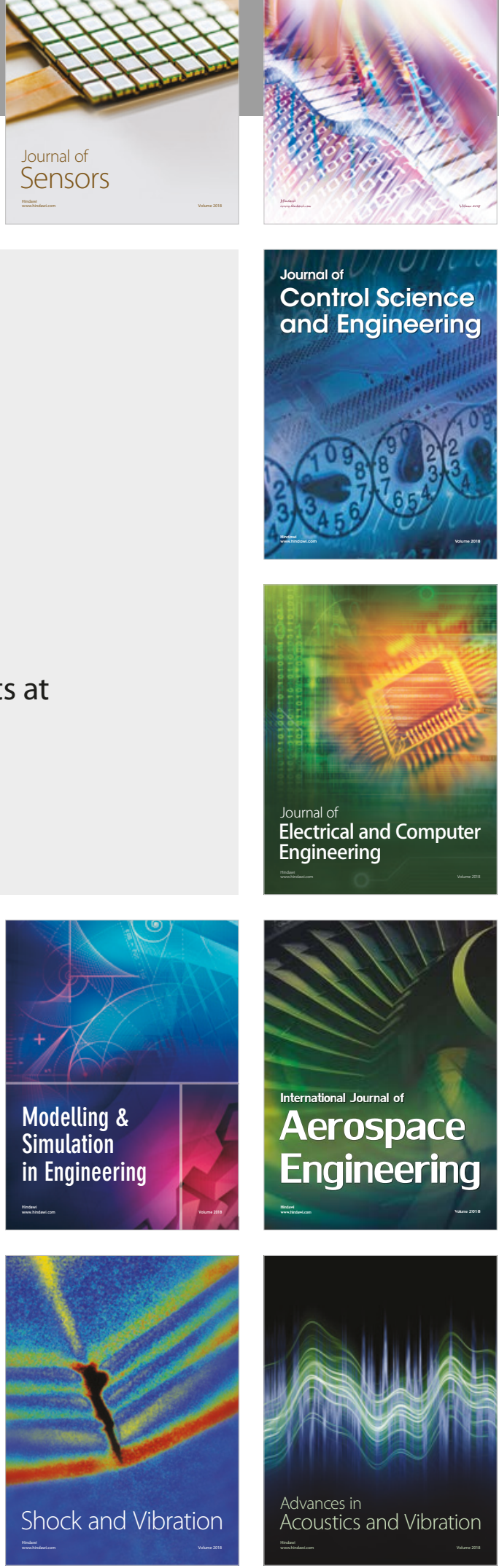